\title{
Generation of mesoscopic superpositions of two squeezed states of motion for a trapped ion
}

\author{
S.-C. Gou, J. Steinbach, and P. L. Knight \\ Optics Section, Blackett Laboratory, Imperial College, London SW7 2BZ, United Kingdom
}

(Received 20 December 1996)

\begin{abstract}
We propose a scheme to generate mesoscopic superpositions of two squeezed states of motion using a highly anisotropic two-dimensional trap in which the ion's motion is tightly bound in the $Y$ direction. In our scheme, the ion is driven multichromatically by three codirectional laser beams along the $X$ axis, as well as a resonant laser beam along the $Y$ axis. We show that, with the appropriate chosen initial states, the desired states of motion are realized in the $X$ direction when the system reaches its steady state, which is indicated by the extinction of the fluorescence from the ion. [S1050-2947(97)00405-8]
\end{abstract}

PACS number(s): 42.50.Vk, 42.50.Dv, 32.80.Pj

The generation of nonclassical states of light is at the heart of quantum optics. In particular, the squeezed states of light, with reduced noise in one quadrature below the vacuum level, have been extensively studied in the past two decades [1]. A squeezed state is defined as

$$
|\alpha, \xi\rangle=D(\alpha) S(\xi)|0\rangle
$$

where $|0\rangle$ is the vacuum state, $D(\alpha)=\exp \left(\alpha a^{\dagger}-\alpha^{*} a\right)$ is the displacement operator, and $S(\xi)=\exp \left[\left(\xi^{*} a^{2}-\xi a^{\dagger 2}\right) / 2\right]$ is the "squeeze" operator, with the squeezing factor $\xi=r \exp (i \theta)$. Here $a$ and $a^{\dagger}$ are the annihilation and creation operators for the harmonic oscillator, respectively. Defining the two quadrature phase operators $X_{1,2}$ by $X_{1}=a^{\dagger} e^{i \theta / 2}+a e^{-i \theta / 2}$ and $X_{2}=i\left(a^{\dagger} e^{i \theta / 2}-a e^{-i \theta / 2}\right)$, it follows that the variances of $X_{1,2}$ given by Eq. (1) satisfy the minimum uncertainty relation $\left(\Delta X_{1}\right)^{2}\left(\Delta X_{2}\right)^{2}=1$, with $\left(\Delta X_{1}\right)^{2}=e^{-2 r}$ and $\left(\Delta X_{2}\right)^{2}=e^{2 r}$, i.e., the fluctuations in one quadrature are exponentially reduced at the expense of exponentially increased uncertainty in the other.

In addition to the squeezed states, macroscopic superpositions of coherent states are also considered as an important type of nonclassical quantum states [2]. The archetype of such classes of states are the even and odd coherent states, which are described as $\left|\beta_{ \pm}\right\rangle=N_{ \pm}(|\beta\rangle \pm|-\beta\rangle)$, where $|\beta\rangle$ is a coherent state and $N_{ \pm}=\left(2 \pm 2 e^{-2|\beta|^{2}}\right)^{-1 / 2}$ are the normalization constants. For $|\beta| \gg 1$, such states can be visualized as superpositions of two macroscopically distinguishable states, the so-called Schrödinger cat states. These states are of particular interest because they possess various nonclassical properties, such as squeezing and sub-Poissonian statistics despite being made up of quantum states having the strongest classical analog. In this paper, we propose a scheme to prepare superpositions of two squeezed states of motion in an ion trap, which are given in the form, $\left|\varphi_{ \pm}\right\rangle$ $\propto(|\alpha, \xi\rangle \pm|-\alpha, \xi\rangle)$, where $|\alpha, \xi\rangle$ is the squeezed state defined in Eq. (1). These superposed states are referred to as even and odd squeezed states [3] that can be formulated in analogy with the even and odd coherent states. It is well known that the states $\left|\beta_{ \pm}\right\rangle$are eigenstates of the operator $a^{2}$, i.e.,

$$
a^{2}\left|\beta_{ \pm}\right\rangle=\beta^{2}\left|\beta_{ \pm}\right\rangle \text {. }
$$

Applying the squeeze operator $S(\xi)$ on both sides of Eq. (2), it is easy to show that

$$
\left[\cosh (r) a+\sinh (r) e^{i \theta} a^{\dagger}\right]^{2}\left|\varphi_{ \pm}\right\rangle=\beta^{2}\left|\varphi_{ \pm}\right\rangle,
$$

provided that $\alpha=\cosh (r) \beta-\sinh (r) e^{i \theta} \beta^{*}$. Equation (3) can be explicitly expressed as

$$
\begin{gathered}
{\left[a^{2}+2 \tanh (r) e^{i \theta} a^{\dagger} a+\tanh ^{2}(r) e^{2 i \theta} a^{\dagger 2}\right]\left|\varphi_{ \pm}\right\rangle} \\
=\left[\left(\frac{\beta}{\cosh (r)}\right)^{2}-\tanh (r) e^{i \theta}\right]\left|\varphi_{ \pm}\right\rangle,
\end{gathered}
$$

in which various two-quantum processes are involved. The realization of such states is of fundamental importance as they demonstrate the quantum interference effects generated by two already nonclassical states [4] rather than the interference between two quasiclassical states. The problem remains of how such states can be generated and in this paper we address this issue.

Recently, due to the remarkable advances in laser cooling of trapped ions [5], it has become possible to realize nonclassical states of the center-of-mass motion of a single trapped ion. When an ion is trapped in a harmonic potential and driven by external laser beams, its internal and external degree of freedom are coupled via the momentum exchange between the field and the ion [6]. Thus one can manipulate the external motion of the ion by controlling the configuration of the driving lasers. Dissipative effects for the ion motion can be significantly suppressed as the coupling between the vibrational modes and the environment is extremely weak. This unique feature thus makes trapped ions a versatile tool to examine fundamental aspects of quantum mechanics, such as generation of nonclassical states of motion [7-10], the implementation of quantum logic gates [11], and quantum state reconstruction $[12,13]$.

Consider the quantized motion of a two-level ion of mass $M$ trapped in an anisotropic two-dimensional (2D) harmonic potential characterized by the vibration frequencies $\nu_{x}$ and $\nu_{y}$ in the $X$ and $Y$ direction, respectively. The annihilation (creation) of vibrational quanta in the $X$ and $Y$ directions is described by the operators $a \quad\left(a^{\dagger}\right)$ and $b \quad\left(b^{\dagger}\right)$, respectively. Accordingly, the position operators are given by $x=\sqrt{1 / 2 \nu_{x} M}\left(a+a^{\dagger}\right)$ and $y=\sqrt{1 / 2 \nu_{y} M}\left(b+b^{\dagger}\right)$ (we set $\hbar=1$ throughout this paper). In our scheme, four laser beams 
are required to manipulate the motion of the ion: the first three laser beams whose detuning are set to 0 , and $\pm 2 \nu_{x}$, respectively, are applied along the $X$ axis; the fourth laser beam is in resonance with the atomic transition and excites the ion along the $Y$ axis. In the rotating-wave approximation, the Hamiltonian for this system is given by

$$
H=\nu_{x} a^{\dagger} a+\nu_{y} b^{\dagger} b+\frac{\omega_{0}}{2} \sigma_{z}+\left[\mathcal{D} E^{(-)}(x, y, t) \sigma_{-}+\text {H.c. }\right]
$$

where the first three terms in Eq. (5) describe the free evolution of external and internal degrees of freedom of the ion and the last indicates the atom-field interaction. The atomic operators are defined as $\sigma_{z}=|e\rangle\langle e|-| g\rangle\left\langle g\left|, \sigma_{+}=\right| e\right\rangle\langle g|$ and $\sigma_{-}=|g\rangle\langle e|$, where $|e\rangle$ and $|g\rangle$ are the atomic excited and ground states, respectively. The transition in the two-level ion is characterized by the dipole matrix element $\mathcal{D}$ and the transition frequency $\omega_{0}$. The negative frequency part of the classical driving electric field is given by

$$
\begin{aligned}
E^{(-)}(x, y, t)= & E_{0} e^{i\left[\omega_{0} t-k_{0} x+\phi_{0}\right]}+E_{1} e^{i\left[\left(\omega_{0}-2 \nu\right) t-k_{1} x+\phi_{1}\right]} \\
& +E_{2} e^{i\left[\left(\omega_{0}+2 \nu\right) t-k_{2} x+\phi_{2}\right]}+E_{3} e^{i\left[\omega_{0} t-k_{0} y+\phi_{3}\right]},
\end{aligned}
$$

where $E_{j}$ and $\phi_{j}$ indicate the amplitudes and phases of the driving lasers, respectively.

Vogel and de Matos Filho $[8,14]$ have pointed out that in the resolved sideband limit where the trapping frequency is much larger than the other characteristic frequencies, the ionlaser interaction can be described as a nonlinear JaynesCummings model, provided that the driving laser is resonant with one of the vibrational sidebands. Following the approach of Refs. [8,14], the Hamiltonian of Eq. (5) can be expressed in the interaction picture as

$$
\begin{aligned}
H_{I}= & \left\{e ^ { - \eta _ { x } ^ { 2 } / 2 } \sum _ { j = 0 } ^ { \infty } \frac { ( i \eta _ { x } ) ^ { 2 j + 2 } } { j ! ( j + 2 ) ! } \left[\Omega_{1} e^{i \phi_{1}} a^{\dagger j+2} a^{j}\right.\right. \\
& \left.+\Omega_{2} e^{i \phi_{2}} a^{\dagger j} a^{j+2}\right]+e^{-\eta_{x}^{2} / 2} \sum_{j=0}^{\infty} \frac{\left(i \eta_{x}\right)^{2 j}}{j ! j !} \Omega_{0} e^{i \phi_{0}} a^{\dagger j} a^{j} \\
& \left.+e^{-\eta_{y}^{2} / 2} \sum_{j=0}^{\infty} \frac{\left(i \eta_{y}\right)^{2 j}}{j ! j !} \Omega_{3} e^{i \phi_{3}} b^{\dagger j} b^{j}\right\} \sigma_{-}+\text {H.c. },
\end{aligned}
$$

where $\Omega_{j}=\mathcal{D} E_{j}$ are the Rabi frequencies and the LambDicke parameters $\eta_{x}=\sqrt{k_{L}^{2} / 2 M \nu_{x}}, \quad \eta_{y}=\sqrt{k_{L}^{2} / 2 M \nu_{y}}$ are defined assuming $k_{0} \simeq k_{1} \simeq k_{2}=k_{L}$.

As the damping of vibrational quanta can be significantly suppressed in an ion trap, the dominant decay process is spontaneous emission from the two-level ion and the time evolution of the system in the interaction picture can be described by a density operator $\rho$ obeying the master equation [8]

$$
\frac{d \rho}{d t}=-i\left[H_{I}, \rho\right]+\frac{\Gamma}{2}\left(2 \sigma_{-} \tilde{\rho} \sigma_{+}-\sigma_{+} \sigma_{-} \rho-\rho \sigma_{+} \sigma_{-}\right),
$$

where $\Gamma$ is the spontaneous decay rate of the excited state of the ion, and the modified density operator

$$
\tilde{\rho}=\frac{1}{4} \int_{-1}^{1} \int_{-1}^{1} d u d v \mathcal{A}(u, v) e^{i k_{L}(u x+v y)} \rho e^{-i k_{L}(u x+v y)}
$$

accounts for the momentum transfer in the $X-Y$ plane due to spontaneous emission described by the angular distribution $\mathcal{A}(u, v)$. In this paper, we consider the case where the ion is confined in a highly anisotropic 2D trap with $\nu_{x} \ll \nu_{y}[15]$, i.e., the ion is tightly bound in the $Y$ direction. In the LambDicke limit where the vibrational amplitude of the ion is much smaller than the laser wavelength, the anisotropy of trapping frequencies leads to the criteria $\eta_{y} \ll \eta_{x} \ll 1$, and thus the master equation, Eq. (8), can be well approximated by expansion of $\eta_{x}$ and $\eta_{y}$ to the first few lowest orders. In particular, we assume that $\mathcal{A}(u, v)$ is an even function of $u$ and $v$ and that all driving fields are of the same order of magnitude. It is thus sufficient to expand the Hamiltonian $H_{I}$ to the second order of $\eta_{x}$ and zeroth order of $\eta_{y}$ so that the master equation Eq. (8) is simplified to

$$
\begin{aligned}
\frac{d \rho}{d t}= & -i\left[H_{I}^{\prime}, \rho\right]+\frac{\Gamma}{2}\left(2 \sigma_{-} \rho \sigma_{+}-\sigma_{+} \sigma_{-} \rho-\rho \sigma_{+} \sigma_{-}\right) \\
& +O\left(\Gamma \eta_{x}^{2}\right)
\end{aligned}
$$

where $H_{I}^{\prime}$ is the effective Hamiltonian

$$
\begin{aligned}
H_{I}^{\prime}= & \left(-g_{1} e^{-i \phi_{1}} a^{2}-g_{2} e^{-i \phi_{2}} a^{\dagger 2}-2 g_{0} e^{-i \phi_{0}} a^{\dagger} a+\zeta\right) \sigma_{+} \\
& + \text {H.c., }
\end{aligned}
$$

with $g_{j}=\eta_{x}^{2} \Omega_{j} / 2$ and $\zeta=\Omega_{0} e^{-i \phi_{0}}+\Omega_{3} e^{-i \phi_{3}}$. Here we have ignored the exponential factors in Eq. (7), as the small Lamb-Dicke parameters $\eta_{y} \ll \eta_{x} \ll 1$ imply that $\exp \left(-\eta_{x}^{x} / 2\right) \simeq \exp \left(-\eta_{y}^{2} / 2\right)=1$. In order for the approximated Hamiltonian Eq. (11) to be valid, we also need to choose appropriate field and phase, i.e., $E_{3}$ and $\phi_{3}$, such that $|\zeta|$ has the same order of magnitude as $g_{j}$, i.e., the field along the $Y$ axis is utilized to balance the overwhelmingly large contribution from the zeroth-order expansion of $\eta_{x}$ in the $X$ direction. It should be noted that if the higher-order terms are important, the desired steady vibrational states would never be reached. Therefore, it is significant to assume a small Lamb-Dicke parameter in our scheme. Nevertheless, we note that $\eta_{x}$ cannot be too small in real experiments, for the finite size of $\eta_{x}$ is necessary to allow the second sideband excitation. Typically, a small Lamb-Dicke parameter with order of magnitude ranging from $10^{-1}$ from $10^{-2}$ can be demonstrated in experiments $[12,13]$. If we further assume that $\Gamma \ll \Omega_{j}$, then the high-order terms $O\left(\Gamma \eta_{x}^{2}\right)$ in Eq. (10) can be neglected and thus the steady-state solution $\rho_{\mathrm{ss}}$ for the master equation is given by

$$
\rho_{s s}=|g\rangle\langle g| \otimes \rho_{v s}
$$

as a consequence of the atomic spontaneous decay, where $\rho_{v s}$ is the vibrational steady state of the ion. If we substitute 
the steady state $\rho_{s s}$ into the equation $d \rho_{s s} / d t=0$, we find the necessary condition $\left[H_{I}^{\prime}, \rho_{s s}\right]=0$, or, equivalently,

$$
\left(g_{1} e^{-i \phi_{1}} a^{2}+g_{2} e^{-i \phi_{2}} a^{\dagger 2}+2 g_{0} e^{-i \phi_{0}} a^{\dagger} a\right) \rho_{v s}=\zeta \rho_{v s} .
$$

The operator on the left-hand side of Eq. (13) is equivalent to that on the left-hand side of Eq. (4) apart from an overall scaling, provided that the configuration of the driving lasers along the $X$ direction is specified by $E_{0} / E_{1}=\tanh (r)$, $E_{2} / E_{1}=\tanh ^{2}(r)$ and $\theta=\phi_{1}-\phi_{0}, 2 \theta=\phi_{1}-\phi_{2}$. Accordingly, the complex eigenvalue $\beta^{2}$ is given by

$$
\frac{\beta^{2}}{\cosh ^{2}(r)}=\frac{2}{\eta_{x}^{2}}\left[\frac{E_{0}}{E_{1}} e^{i \theta}+\frac{E_{3}}{E_{1}} e^{i\left(\phi_{1}-\phi_{3}\right)}\right]+\frac{E_{0}}{E_{1}} e^{i \theta},
$$

which is determined by the two resonant fields applied along the $X$ and $Y$ axes, respectively. We note that, since $\tanh (r)$ $\rightarrow 1$ as $r \rightarrow \infty$, we require $E_{1}>E_{0}>E_{2}$, to guarantee the existence of a normalized steady state of motion.

In general, the vibrational steady-state solution $\rho_{v s}$ is not uniquely determined by Eq. (13). In order to ensure the steady-state solution approaches the even and odd squeezed states, additional restrictions on the initial vibrational states are required. As the time evolution described by $H_{I}^{\prime}$ does not mix even and odd states, an arbitrary initial state $|\varphi(0)\rangle=\sum_{n=0}^{\infty} c_{n}|n\rangle$ can be separated into states having opposite parities, i.e., with

$$
\left|\chi_{e}\right\rangle=\sum_{n=0}^{\infty} c_{2 n}|2 n\rangle
$$

being the state of even parity and

$$
\left|\chi_{o}\right\rangle=\sum_{n=0}^{\infty} c_{2 n+1}|2 n+1\rangle
$$

being the state of odd parity. For any initial vibrational state having a mixed parity, the steady-state solution of Eq. (13) is given by a statistical mixture of even and odd squeezed states

$$
\rho_{v s}=P_{e}\left|\varphi_{+}\right\rangle\left\langle\varphi_{+}\left|+P_{o}\right| \varphi_{-}\right\rangle\left\langle\varphi_{-}\right|,
$$

where $P_{e}$ and $P_{o}$ are the weights of the even and odd parity states in the initial state. However, if the initial state is prepared in a precise parity, either even or odd, then the steady state of motion is a pure state. It is straightforward to verify that if the initial state of motion is given by $\left|\chi_{e}\right\rangle\left(\left|\chi_{o}\right\rangle\right)$, then as $t \rightarrow \infty$ the system evolves to $\left|\varphi_{+}\right\rangle\left(\left|\varphi_{-}\right\rangle\right)$in which only quanta of even (odd) numbers exist. For simplicity, $\left|\chi_{e}\right\rangle$ and $\left|\chi_{o}\right\rangle$ can be prepared in the Fock state $|0\rangle$ and $|1\rangle$ which, according to recent experiments, can be prepared with very high efficiency $[10,13]$.

In order to check the validity of our analytic argument, we have employed a Monte Carlo state-vector method [16] to solve the master equation without using a Lamb-Dicke expansion. Our numerical analysis was performed using a delay function technique [17] in a truncated Fock-state basis with a cutoff chosen such that an increase of this cutoff does not alter the result of our integration. The vibrational number

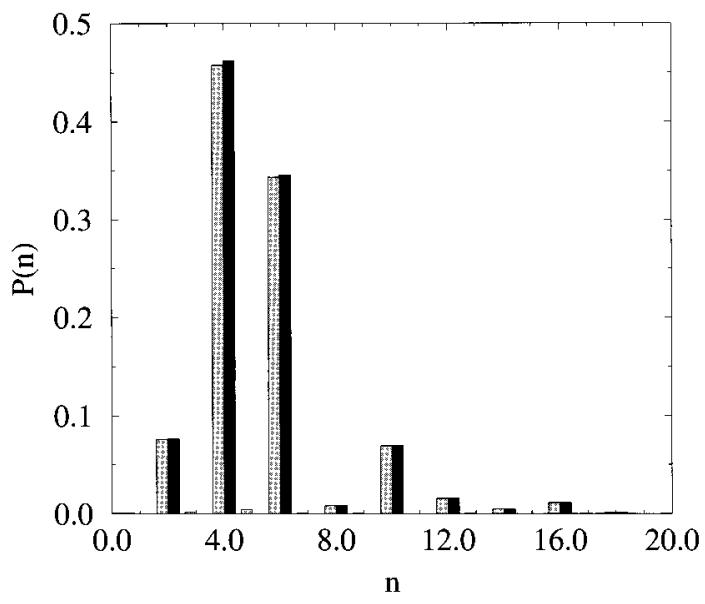

FIG. 1. The number distribution $P(n)$ of the vibrational steady state (gray bars) for the ion initially prepared in the vacuum state and the Lamb-Dicke parameter $\eta_{x}=0.02 ; \operatorname{Tr}\left(\rho_{\mathrm{vs}}^{2}\right)=0.98$. The number distribution of the even squeezed state, $\alpha=2$ and $\xi=1$, are shown in dark bars.

distribution $P(n)$ for the ion in its steady state is shown in Fig. 1, which is obtained by numerically integrating the master equation containing all powers of a small Lamb-Dicke parameter $\eta_{x}=0.02$ with the initial vibrational states prepared in the vacuum state. It is seen that the number distribution is in good agreement with that of $\left|\varphi_{+}\right\rangle$with a negligible difference caused by other higher-order terms that have been ignored in the analytical treatment. The purity of the steady state in Fig. 1 is indicated by $\operatorname{Tr}\left(\rho_{v s}^{2}\right)=0.98$ that is very close to a pure state. In Fig. 2, we show the number distribution of the steady state given by a larger Lamb-Dicke parameter $\eta_{x}=0.05$. In this case, the purity of the steady state is given by $\operatorname{Tr}\left(\rho_{v s}^{2}\right)=0.88$ indicating an appreciable difference from the desired pure state, as can be seen from the number distribution in Fig. 2. The Wigner distribution function of the even and odd squeezed states are shown in Figs. 3 and 4 . In comparison with those given by the even and odd coherent states [8], the fringes of quantum interference are more pronounced. The nonclassical nature of the even and odd squeezed states is indicated by the negative values of the Wigner distribution function.

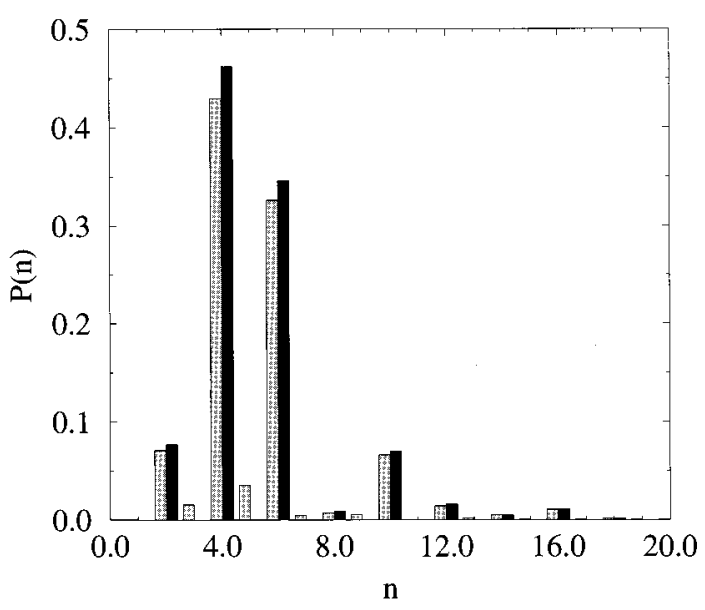

FIG. 2. The same as Fig. 1 except $\eta_{x}=0.05 ; \operatorname{Tr}\left(\rho_{v s}^{2}\right)=0.88$. 


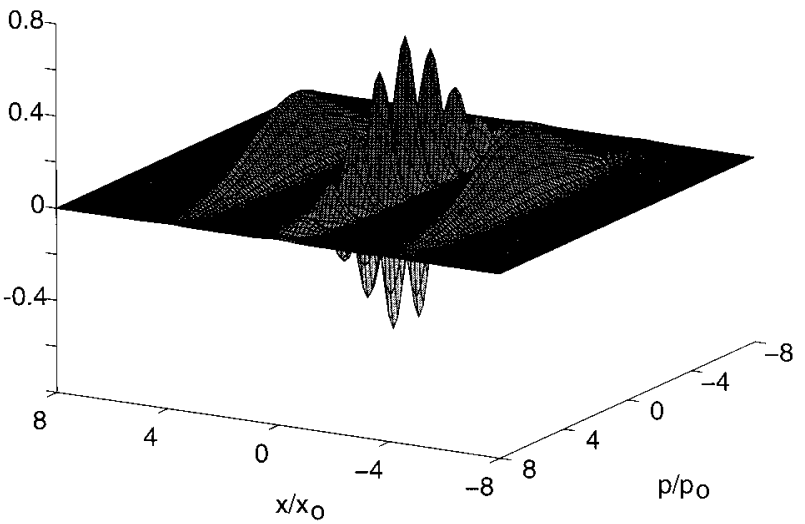

FIG. 3. The Wigner distribution function for the even squeezed states, $\alpha=2$ and $\xi=1$, with the scaling factors $x_{0}$ $=\left(2 \nu_{x} M\right)^{-1 / 2}, p_{0}=\left(\nu_{x} M / 2\right)^{-1 / 2}$.

We noticed that in a very recent paper [3] Nieto has studied the dynamical behavior of the spatial probability distribution of even and odd squeezed states. The spatial distribution consists of two squeezed wave packets which are phase separated by $\pi$ with respect to their coherent displacements at $t=0$, evolving periodically with a period $2 \pi / \nu_{x}$. The quantum interferences appear when the two wave packets move towards each other at the time $t_{j}=(j+1 / 2) \pi / \nu_{x}$, where $j$ is an integer. It has been shown that if the initial separated wave packets have a small $x$ uncertainty, then the colliding wave packets at $t_{j}$ have a large $x$ uncertainty and exhibit a broad and very pronounced interference pattern symmetric with respect to the origin. On the other hand, however, if the wave packets have large $x$ uncertainty initially, the interference pattern at $t_{j}$ shows a highly confined peak structure about the origin, which has a smaller width than that given by the coherent states [8].

In conclusion, we have proposed a scheme for the realiza-

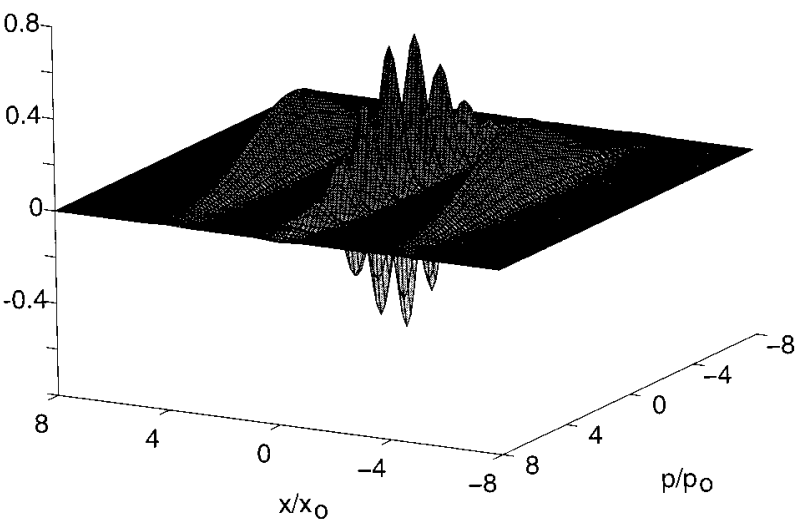

FIG. 4. The Wigner distribution function for the odd squeezed states, $\alpha=2$ and $\xi=1$ with the scaling factors $x_{0}$ $=\left(2 \nu_{x} M\right)^{-1 / 2}, p_{0}=\left(\nu_{x} M / 2\right)^{-1 / 2}$.

tion of even and odd squeezed states of the center-of-mass motion of the trapped ion. In our scheme, the ion which is trapped in a highly anisotropic 2D harmonic potential well is driven multichromatically by four laser beams. Three laser beams with detunings $\Delta=0, \pm 2 \nu_{x}$, determining the squeezing factor $\xi$, excite the ion along the $X$ axis; the laser beam with $\Delta=0$ is applied along the $Y$ axis to balance the carrier field along the $X$ axis to generate a moderate displacement $\alpha$. In appropriate limits, the system relaxes to a steady state due to the spontaneous emission from the ion, and can be described as a product of the atomic ground state with a quantum superposition of two squeezed states of motion.

This work was supported in part by the UK Engineering and Physical Sciences Research Council and the European Union. S.-C. Gou is supported by the Ya-Chih Tsai Research Foundation. J. Steinbach is supported by the German Academic Exchange Service (DAAD-Doktorandenstipendium aus Mitteln des zweiten Hochschulsonderprogramms).
[1] H. P. Yuen, Phys. Rev. A 13, 2226 (1976); R. Loudon and P. L. Knight, J. Mod. Opt. 34, 709 (1987).

[2] For a recent review and many references, see V. Bužek and P. L. Knight, in Progress in Optics, edited by E. Wolf (Elsevier, Amsterdam, 1995), Vol. XXXIV.

[3] M. M. Nieto, Phys. Lett. A 219, 180 (1996).

[4] The nonclassical properties of the superpositions of two squeezed vacuum states have been examined by B. C. Sanders, see B. C. Sanders, Phys. Rev. A 39, 4284 (1989).

[5] F. Diedrich, J. C. Bergquist, W. M. Itano, and D. J. Wineland, Phys. Rev. Lett. 62, 403 (1989); C. Monroe, D. M. Meekhof, B. E. King, S. R. Jefferts, W. M. Itano, and D. J. Wineland, Phys. Rev. Lett. 75, 4011 (1995).

[6] C. A. Blockley, D. F. Walls, and H. Risken, Europhys. Lett. 17, 509 (1992); C. A. Blockley and D. F. Walls, Phys. Rev. A 47, 2115 (1993).

[7] J. I. Cirac, R. Blatt, A. S. Parkins, and P. Zoller, Phys. Rev. Lett. 70, 762 (1993); J. I. Cirac, A. S. Parkins, R. Blatt, and P. Zoller, ibid. 70, 556 (1993).
[8] R. L. de Matos Filho and W. Vogel, Phys. Rev. Lett. 76, 608 (1996).

[9] S.-C. Gou, J. Steinbach, and P. L. Knight, Phys. Rev. A 54, R1014 (1996).

[10] D. M. Meekhof, C. Monroe, B. E. King, W. M. Itano, and D. J. Wineland, Phys. Rev. Lett. 76, 1796 (1996); C. Monroe, D. M. Meekhof, B. E. King, and D. J. Wineland, Science 272, 1131 (1996).

[11] C. Monroe, D. M. Meekhof, B. E. King, W. M. Itano, and D. J. Wineland, Phys. Rev. Lett. 75, 4714 (1995).

[12] S. Wallentowitz and W. Vogel, Phys. Rev. Lett. 75, 2932 (1995); J. F. Poyatos, R. Walser, J. I. Cirac, and P. Zoller, Phys. Rev. A 53, R1966 (1996); C. D’Helon and G. J. Miburn, ibid. 54, R25 (1996).

[13] D. Leibfried, D. M. Meekhof, B. E. King, C. Monroe, W. M. Itano, and D. J. Wineland, Phys. Rev. Lett. 77, 4281 (1996).

[14] W. Vogel and R. L. de Matos Filho, Phys. Rev. A 52, 4214 (1995).

[15] C. C. Gerry, Phys. Rev. A 55, 2478 (1997). 
[16] J. Dalibard, Y. Castin, and K. Mølmer, Phys. Rev. Lett. 68, 580 (1992); K. Mølmer, Y. Castin, and J. Dalibard, J. Opt. Soc. Am. B 10, 542 (1993); H. J. Carmichael, An Open System Approach to Quantum Optics, Lecture Notes in Physics (Springer-Verlag, Berlin, 1993); R. Dum, P. Zoller, and H. Ritsch, Phys. Rev. A 45, 4879 (1992); G. C. Hegerfeldt and T.
S. Wilser, in Proceedings of the Second International Wigner Symposium, Goslar, 1991, edited by H. D. Doebner, W. Scherer, and F. Schroeck (World Scientific, Singapore, 1992).

[17] R. Dum, A. S. Parkins, P. Zoller, and C. W. Gardiner, Phys. Rev. A 46, 4382 (1992). 